\title{
Bifurcation and Stability Properties of Periodic Solutions to Two Nonlinear Spring-Mass Systems
}

\author{
N. Ben-Gal * K.S. Moore ${ }^{\dagger}$
}

May 2, 2006

\begin{abstract}
The behavior of a periodically forced, linearly damped mass suspended by a linear spring is well-known. In this paper we study the nature of periodic solutions to two nonlinear spring-mass equations; our nonlinear terms are similar to earlier models of motion in suspension bridges. We contrast the multiplicity, bifurcation, and stability of periodic solutions for a piecewise linear and smooth nonlinear restoring force. We find that while many of the qualitative properties are the same for the two models, the nature of the secondary bifurcations (period-doubling and quadrupling) differs significantly.
\end{abstract}

Keywords: suspension bridge, continuation, bifurcation, stability, periodic solutions, nonlinear spring-mass

AMS subject classification: $34 \mathrm{C} 15,34 \mathrm{C} 25$

\section{Introduction}

In [9], Lazer and McKenna proposed a nonlinear beam equation as a model for vertical oscillations in suspension bridges. They modeled the restoring

*Division of Applied Mathematics, Brown University, Providence, RI 02912, fax: 401 863 1355. email:bengal@dam.brown.edu

${ }^{\dagger}$ Department of Mathematics, University of Michigan, Ann Arbor, MI 48109-1109, fax: 734763 0937. email:ksmoore@umich.edu 
force from the cable as a piecewise linear function of the displacement in order to capture the fact that the suspension cables resist elongation, but do not resist compression.

Later investigations of the qualitative and quantitative properties of solutions to this type of asymmetric system suggest that this is a convincing model for nonlinearly suspended structures. The results on existence, uniqueness, multiplicity, bifurcation, and stability of periodic solutions are consistent with the nonlinear behavior of some suspension bridges; see [2], [4], [6], [8], and [13], for example.

In [10] and [14], McKenna and Moore extended the models of Lazer and McKenna to the coupled vertical and torsional motions of suspension bridges. Though they were able to replicate the phenomena observed on the Tacoma Narrows Bridge on the day of its famous collapse [15], the model had several shortcomings. First, the treatment of the restoring force from the cables was oversimplified; the nonlinear terms in the model describe cables that behave perfectly linearly when in tension (regardless of the size of the oscillation) and that can lose tension completely. Moreover, the parameter values for which they could induce the desired phenomena were physically unreasonable.

In [11], McKenna and O'Tuama proposed a modified nonlinearity that addressed the shortcomings described above. In [11] and [12], McKenna, O'Tuama, and Moore found that smoothing the nonlinearity yields a significant qualitative change in the structure of the set of periodic solutions to the nonlinearly coupled vertical-torsional system.

In this paper, we explore and isolate the impact of smoothing the nonlinearity by studying a simper mechanical system. We consider a periodically forced, linearly damped mass suspended by a cable. Of course, if the cable were a linear spring, the resulting behavior would be well known, [1]. We consider two nonlinear models for the restoring force from the cable: a piecewise linear and a smooth nonlinear force. We describe both models in Section 2. In Section 3, we employ a numerical continuation algorithm and stability analysis to examine the bifurcation and stability properties of periodic solutions to the piecewise linear model and we contrast these results with the well known behavior of the linear system. In Section 4, we repeat this analysis for the smoothed model and contrast the results for the piecewise linear and smooth nonlinear systems.

In Section 5, we present results on secondary bifurcations; we find solutions whose period is twice or four times the period of the forcing term. 
Figure 1: A mass suspended from a cable

Moreover, we find that there are significant qualitative differences in the nature of the secondary bifurcations for the two models.

Finally, in Section 6, we describe open questions and future work.

\section{The Models}

We begin by considering a mass $m$ suspended by a cable, see Figure 1 .

Let $y$ be the downward displacement of the mass from the cable's natural equilibrium state. Let $z$ be the downward displacement of the mass from the weighted equilibrium point. The two displacements are related by the equation $y=z+L$, where $L=\frac{m g}{K}$, with $g$ being the acceleration of gravity 
and $K$ the spring constant. This relation was derived simply from Hooke's Law. In each model we consider the forces provided by the cable suspending the mass, damping, gravity, and external forcing. We consider three different models: the linear, piecewise linear, and smoothed nonlinear cable forces.

\subsection{Linear cable force}

In the linear model, we take the cable force to be represented by the simple linear Hooke's Law equation $F=-K y$, or in our second coordinate system, $F=-K z-m g$.

This leads to the differential equations

$$
\begin{gathered}
m y^{\prime \prime}=-K y-\delta y^{\prime}+m g+\lambda \sin (\mu t) \\
m z^{\prime \prime}=-K z-\delta y^{\prime}+\lambda \sin (\mu t)
\end{gathered}
$$

where the first term represents the cable force, the second term is damping, the $m g$ term represents the force of gravity and is subsumed into the first term in equation (2), while the final term is the periodic external forcing. $\delta>0$ is the damping coefficient, $\lambda$ is the amplitude of forcing, while $\mu$ is the forcing frequency.

Since both equations are second order linear ordinary differential equations, we know everything there is to know about them, including their solution:

$$
\begin{aligned}
z & =\underbrace{c_{1} e^{-\frac{\delta t}{2 m}} \cos \left(\frac{\sqrt{4 K m-\delta^{2}}}{2 m} t\right)+c_{2} e^{-\frac{\delta t}{2 m}} \sin \left(\frac{\sqrt{4 K m-\delta^{2}}}{2 m} t\right)}_{\text {Homogeneous solution } z_{h}(t)} \\
+ & \underbrace{\left(\frac{-\lambda \delta \mu}{\left(\mu^{2} m-K\right)^{2}+(\delta \mu)^{2}}\right) \cos (\mu t)+\left(\frac{\lambda\left(K-\mu^{2} m\right)}{\left(\mu^{2} m-K\right)^{2}+(\delta \mu)^{2}} \sin (\mu t)\right)}_{\text {Particular solution } z_{p}(t)}
\end{aligned}
$$

The constants $c_{1}$ and $c_{2}$ are determined by the initial position and velocity of the mass. Because of the damping (i.e., because of the $e^{-\frac{\delta t}{2 m}}$ term), we have that $z_{h}(t) \rightarrow 0$ as $t \rightarrow \infty$, therefore the long term response of the linear system is independent of the initial conditions and is driven entirely by the external forcing. 
Of course, bridges are not suspended by linear springs. Moreover, the phenomena observed at Tacoma Narrows suggest a long-term dependence on initial conditions that is not consistent with the linear model [15]. Therefore, in the next two subsections, we describe nonlinear models for the cable force.

\subsection{Piecewise linear cable force}

In this section, motivated by the original Lazer-McKenna model [9], we consider a more realistic cable force by assuming that the cables resist elongation according to Hooke's Law, as we did in the previous model, but do not resist compression. In other words, $F=0$ when the mass goes above the unloaded state $(y \leq 0$ or $z \leq-L)$.

Thus, the force exerted by the cable is

$$
\begin{gathered}
F=\left\{\begin{array}{cc}
-K y & y>0 \\
0 & y \leq 0
\end{array}=-K y^{+}\right. \\
F=\left\{\begin{array}{cc}
-K(z+L) & z>-L \\
0 & z \leq-L
\end{array}=-K(z+L)^{+}\right.
\end{gathered}
$$

and the differential equation becomes

$$
\begin{gathered}
m y^{\prime \prime}=-K y^{+}-\delta y^{\prime}+m g+\lambda \sin (\mu t) \\
m z^{\prime \prime}=-K(z+L)^{+}-\delta z^{\prime}+m g+\lambda \sin (\mu t)
\end{gathered}
$$

We will see in Section 3 that solutions to this model exhibit behavior that is consistent with the nonlinear oscillations observed in suspension bridges. In particular, we find multiple periodic solutions for fixed forcing amplitude and we see that the effect of the initial conditions does not decay over time as in the linear model.

\subsection{Smoothed nonlinear cable force}

There are some inherent flaws in the piecewise model. For one, the nondifferentiability of this cable force makes it an unlikely candidate to describe a physical system. Physical intuition says that the transition of an object from no exertion to the exertion of a noticeable force is not instantaneous, but must be smooth. In addition, we know that cables do exert some resistance 
to compression and that they exert superlinear force when stretched very far. Therefore, we must again change our model to one that addresses these inadequacies.

In this model, motivated by [11] and [12], we construct a smoothed version of the system. We take the following things into account in this construction. First, we want the cable force to be zero when the system is in equilibrium, at $z=0$. Second, for $z=-\infty$ the cable force must only be supplied by gravity. And finally, the slope of the smooth nonlinear force should match the slope of the linear force, i.e. $F^{\prime}=-K$. The cable force satisfies each of these requirements.

$$
F=m g\left(e^{\frac{K z}{m g}}-1\right)
$$

Our differential equation is now

$$
z^{\prime \prime}=-g\left(e^{\frac{K z}{m g}}-1\right)-\left(\frac{\delta}{m}\right)+\left(\frac{\lambda}{m}\right) \sin (\mu t)
$$

Figure 2 depicts all three cable forces together. We can clearly see that the slopes are the same for $z=0$ and that all cable forces are at zero for $z=0$. In Sections 4 and 5 we will examine bifurcations and stability properties of periodic solutions to this equation and contrast them with the results for the piecewise linear model.

\section{Results for the Piecewise Linear Model}

Let us first consider the qualitative properties of periodic solutions to the piecewise nonlinear model. We will study the bifurcation and stability properties of periodic solutions by examining the bifurcation curves generated by numerical continuation on the forcing amplitude $\lambda$. We employed the numerical continuation method as in [7], [5] to generate the bifurcation curves. We also examined the stability of our computed periodic solutions via the usual method: we linearized the system (7) about the computed periodic solution and examined the magnitude of the eigenvalues of the fundamental solution to the linearized system, [3].

Figure 3 shows the amplitude of the periodic solutions to the piecewise linear model for fixed $m, \delta, K$, and $\mu$, as we vary $\lambda$. In this experiment we chose $m=1, \delta=.01, K=\sqrt{2}$, and $\mu=0.9514$, which is 80 percent of the 


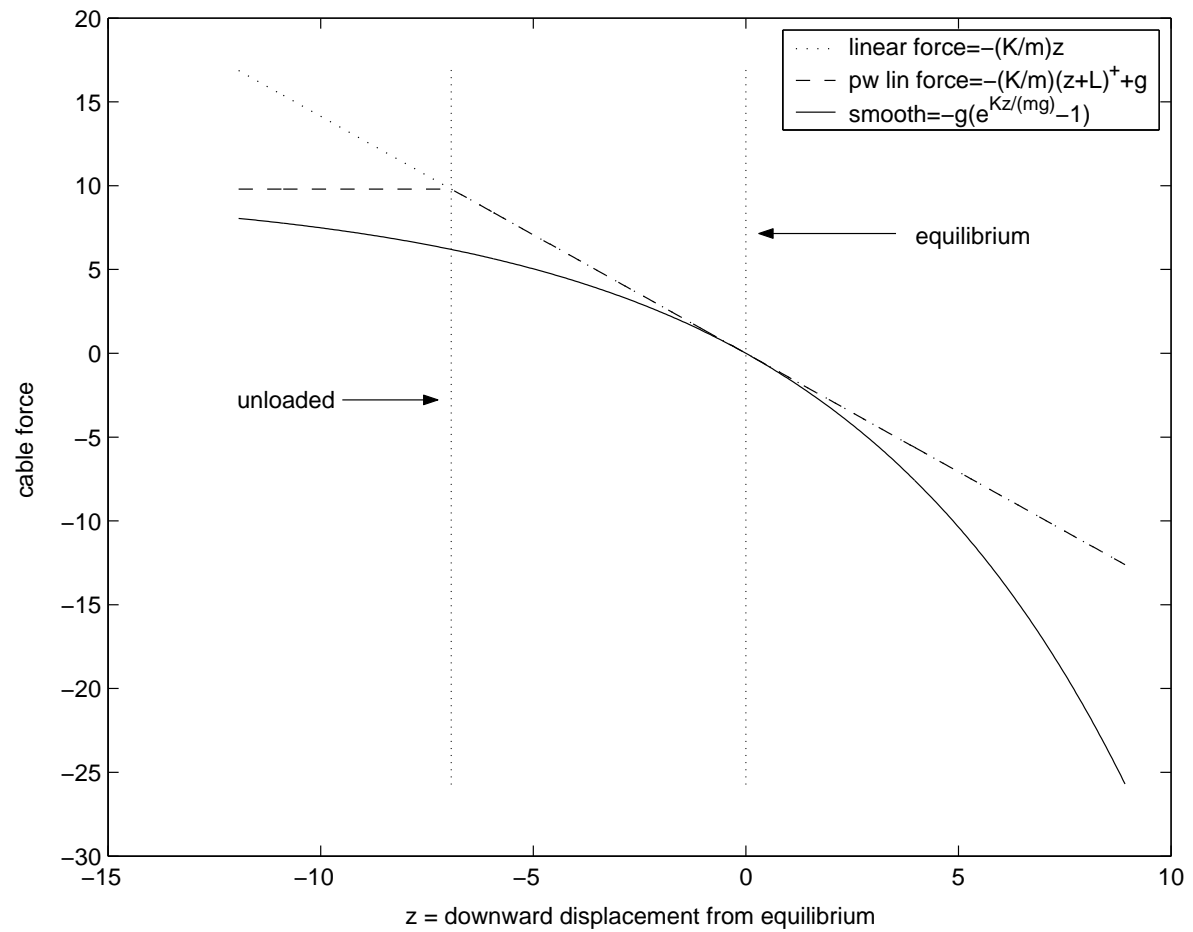

Figure 2: Three different models of the cable force 


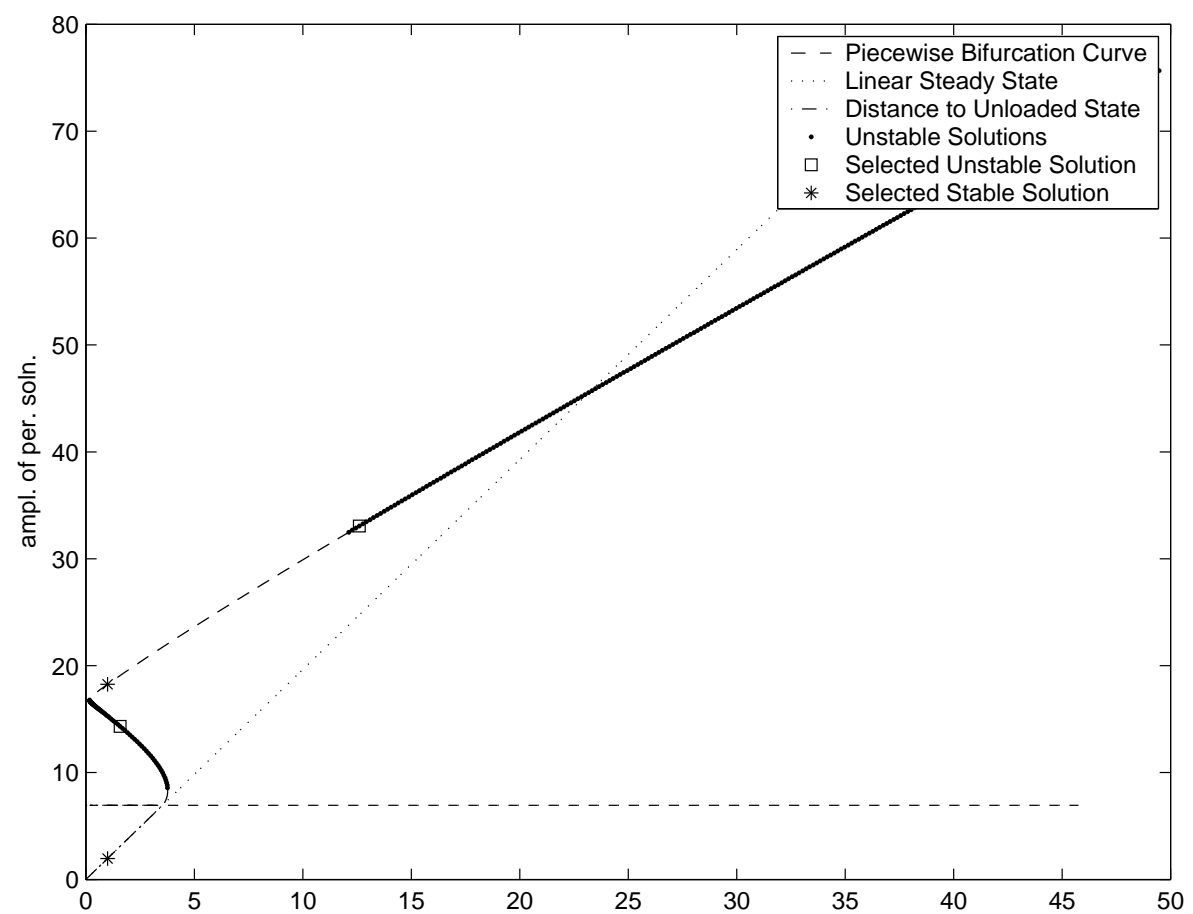

Figure 3: The bifurcation curve of periodic solutions for the piecewise linear model

resonant frequency of the linear system. We see that when $\lambda<\lambda_{l} \approx 0.1667$, there is only one small-amplitude periodic solution. When $\lambda>\lambda_{u} \approx 3.7750$, there is only one large-amplitude solution. When $\lambda \in(0.1667,3.7750)$, there are three distinct periodic solutions. And for $\lambda=\lambda_{l} \approx 0.1667$ or $\lambda=\lambda_{u} \approx$ 3.7750 , i.e. for $\lambda$ at the bifurcation points, there are two distinct periodic solutions. Thus, there is a bifurcation from one to three periodic solutions at $\lambda \approx 0.1667$ and then back to a single periodic solution at $\lambda \approx 3.7750$. Those points on the curve at a particular value of $\lambda$ represent different possible solutions, each with different initial conditions leading to them.

In Figure 3 we see that there is a small periodic solution to the nonlinear equation whose amplitude matches the linear steady state. This is because the amplitude is smaller than $L$; i.e. the mass has not oscillated above the unloaded state and the nonlinearity of the cable force has not come into play. More succinctly, for amplitudes smaller than $L$, when the cable has not become unloaded, the cable behaves exactly like a linear spring; therefore the 


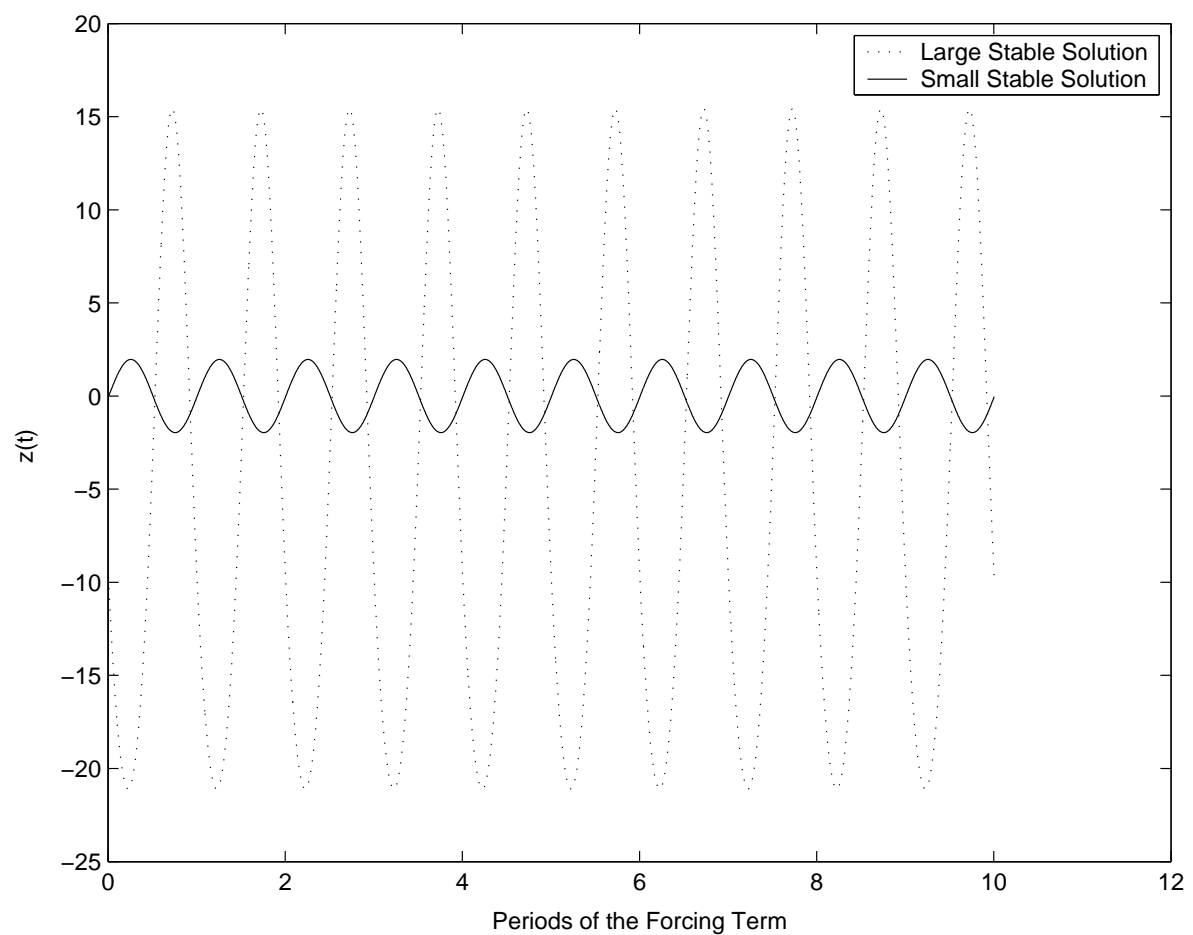

Figure 4 : The same forcing $(\lambda \approx 1.00)$ may induce small or large amplitude oscillations, depending on the initial conditions

periodic solution matches the periodic linear steady state. As we pointed out above, for fixed $\lambda \in(0.1667,3.7750)$, there are two large amplitude solutions in addition to the small amplitude oscillation. For fixed $\lambda$, whether a small, medium, or large amplitude solution results depends only on the initial condition. In Figure 4 we show the stable periodic solutions indicated with an asterisk in Figure 3 . We see that for $\lambda \approx 1.00$, the initial conditions $z(0)=18.2484, z^{\prime}(0)=-9.7461$ yield the large oscillation indicated by the dashed line, while the initial conditions $z(0)=-0.03666, z^{\prime}(0)=1.8695$ yield the small oscillation indicated by the solid line. Because both of these solutions are stable, they will maintain this oscillation indefinitely.

Of course, this behavior could not happen for the linear model. As we pointed out in Section 2.1, in the linear model the effect of the initial conditions decays over time. As we have shown in the experiment above, this is not the case for the nonlinear model; the effect of the initial conditions can persist over large time. 


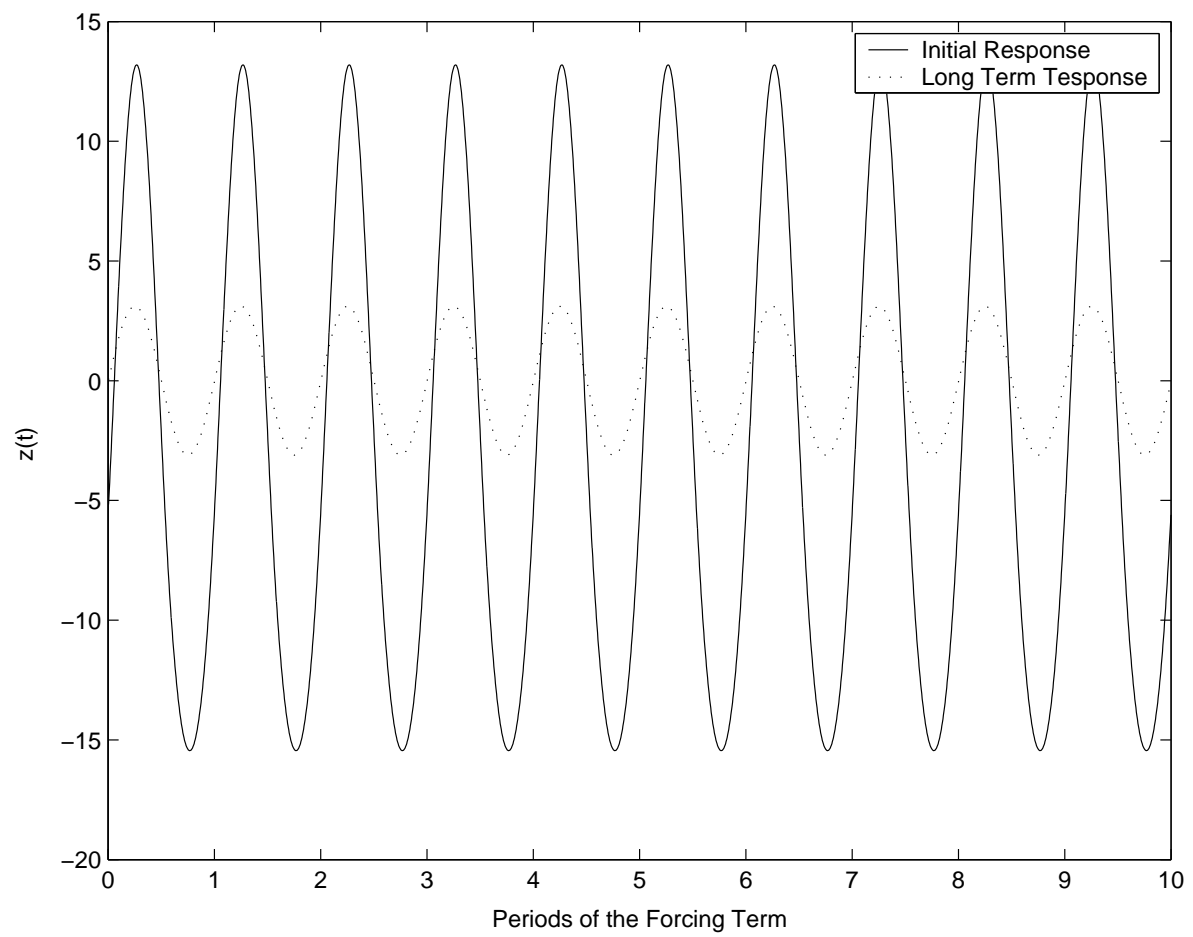

Figure 5: A middle branch unstable solution settles down to small amplitude oscillations

We remark that the solutions pictured are those that we found via numerical continuation; other periodic solutions may exist. Moreover, the bifurcation curve in Figure 3 shows stable and unstable solutions; the unstable solutions are indicated with a dot. The unstable solutions settle down over time to solutions of different amplitude or period. For example, in Figure 5 we show the superposition of periods 1 through 10 and 791 through 800 of the unstable solution indicated with a square on the middle branch of the bifurcation curve in Figure 3.

As you can see, given sufficient time to stabilize, the solution maintains its period, but experiences an approximately 80 percent drop in amplitude, settling to a solution which rests on the lower branch of the bifurcation curve. On the other hand, in Figure 6 we show the same superposition of periods, but this time for the unstable solution indicated with a square on the top branch of the bifurcation curve in Figure 3. In this case, the curve undergoes a doubling of its period, with an alternating 34.9 percent increase and 39.6 


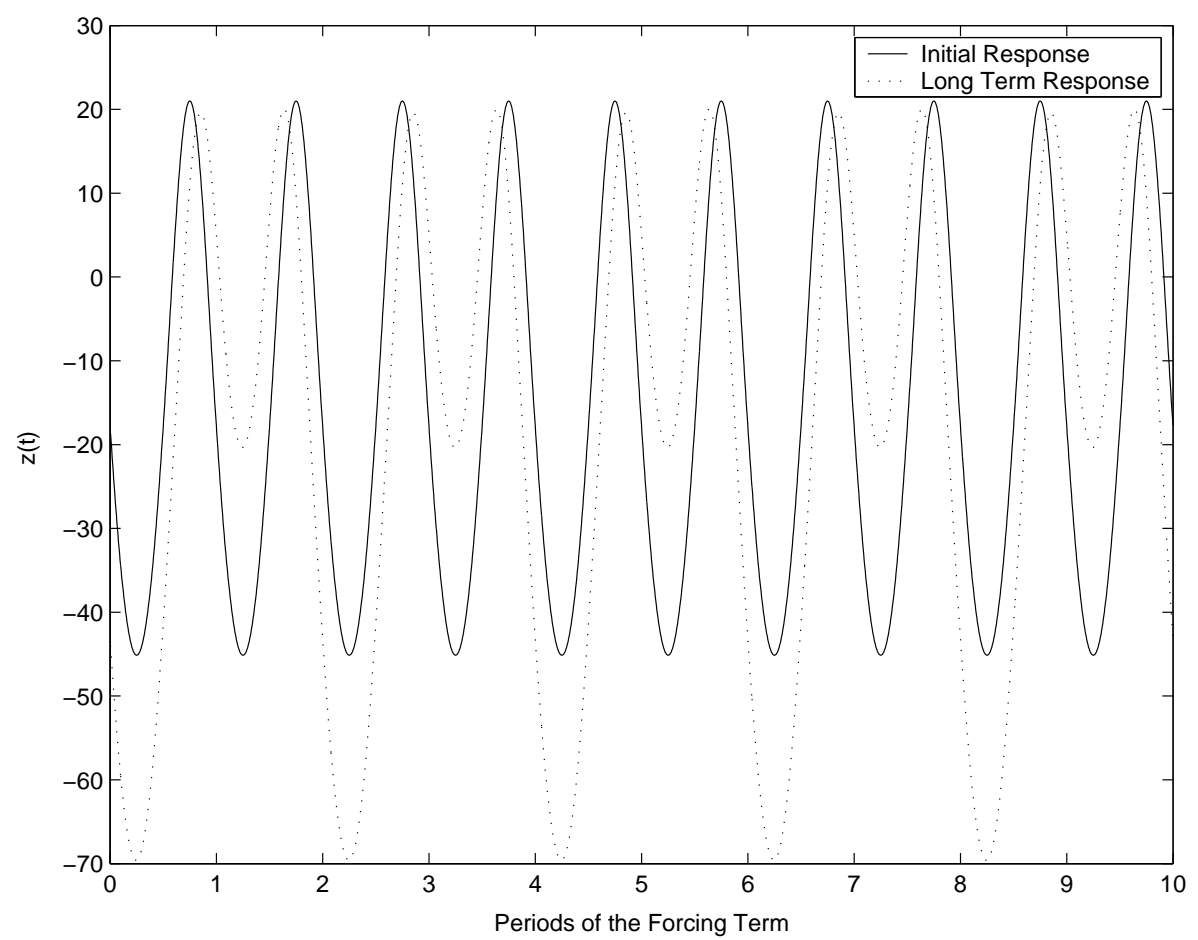

Figure 6: A top branch unstable solution undergoes period doubling with amplitude change

percent reduction in amplitude.

In Section 5, we show that there are secondary bifurcations to branches of solutions with twice or four times the period of the forcing term.

\section{The smoothed model vs. the piecewise lin- ear model: Qualitative differences}

Now that we have examined the bifurcation and stability properties of periodic solutions to the piecewise linear model, the next step is to investigate the qualitative differences between solutions to the smooth nonlinear model and the piecewise linear model. In the experiments that follow, we examine the impact of increasing the forcing frequency toward resonance and of changing the cable tension $K$. More specifically we compute $\mu_{\text {res }}$, the resonant 
frequency for the linear model and examine forcing frequencies $\mu=\eta \mu_{\text {res }}$ for $\eta=0.80,0.90$, and 0.95 . Also, we fix $m=1$ and vary the Hooke's Law constant $K$. When we increase $K$, we are amplifying the asymmetry in the piecewise linear force and changing the curvature in the smoothed force; see Figure 2 in Section 2.3.

\subsection{Impact of varying $\eta$}

There are many qualitative differences that occur when the forcing frequency is increased towards resonance. Figure 7 shows the impact of varying $\eta:=$

$\frac{\mu}{\mu_{r e s}}$, with $m=1, \delta=.01$, and $K=\sqrt{2}$. Figure 8 shows a close-up of Figure 7 near the bifurcation points.

As we increase $\eta$ to 1, i.e. increase the forcing frequency towards resonance, the piecewise bifurcation curve moves above the smooth curve. As you can see in Figure 8, for $\eta=0.8$ the smooth curve is higher than the piecewise for all values of $\lambda$, indicating that for the same value of $\lambda$ the smooth nonlinear solutions are larger than the piecewise solutions. For $\eta=0.9$ the piecewise curve rises above the smooth curve in the middle branch and then crosses below the smooth again. This implies that, for a small range of $\lambda$, the mid-size piecewise solutions are larger than the mid-size smooth solutions. For $\eta=0.95$ the piecewise curve stays above the smooth curve until further into the top branch, and always crosses under after the second bifurcation point. This held for all values of $K$ that we tested. Therefore, for all values of $\lambda$ at which there are three solutions, the mid-size and large solutions of the piecewise model are larger than those of the smooth model.

In addition, as $\eta$ goes to 1 , the values of $\lambda$ at which the curve bifurcates get closer to zero, thus, the bifurcation from single to multiple periodic solutions (and back) occurs for smaller forcing when the frequency is closer to resonance. This occurs for both bifurcation points but the change is much larger for the second bifurcation point. For example, when $\eta=0.80$, we see that for the piecewise curve $\lambda_{l} \approx 0.1667$ and $\lambda_{u} \approx 3.775$, and for the smoothed nonlinear curve $\lambda_{l} \approx .2031$ and $\lambda_{u} \approx 3.414$. But when $\eta=0.95$, for the piecewise curve $\lambda_{l} \approx 0.1084$ and $\lambda_{u} \approx 0.9658$, and for the smoothed nonlinear curve $\lambda_{l} \approx 0.0925$ and $\lambda_{u} \approx 0.4200$.

One effect that occurs in both the smooth and piecewise curves is that the top and middle branches get lower, or in physical terms, as we get closer to resonance the amplitudes of oscillation get smaller for all periodic solutions. 

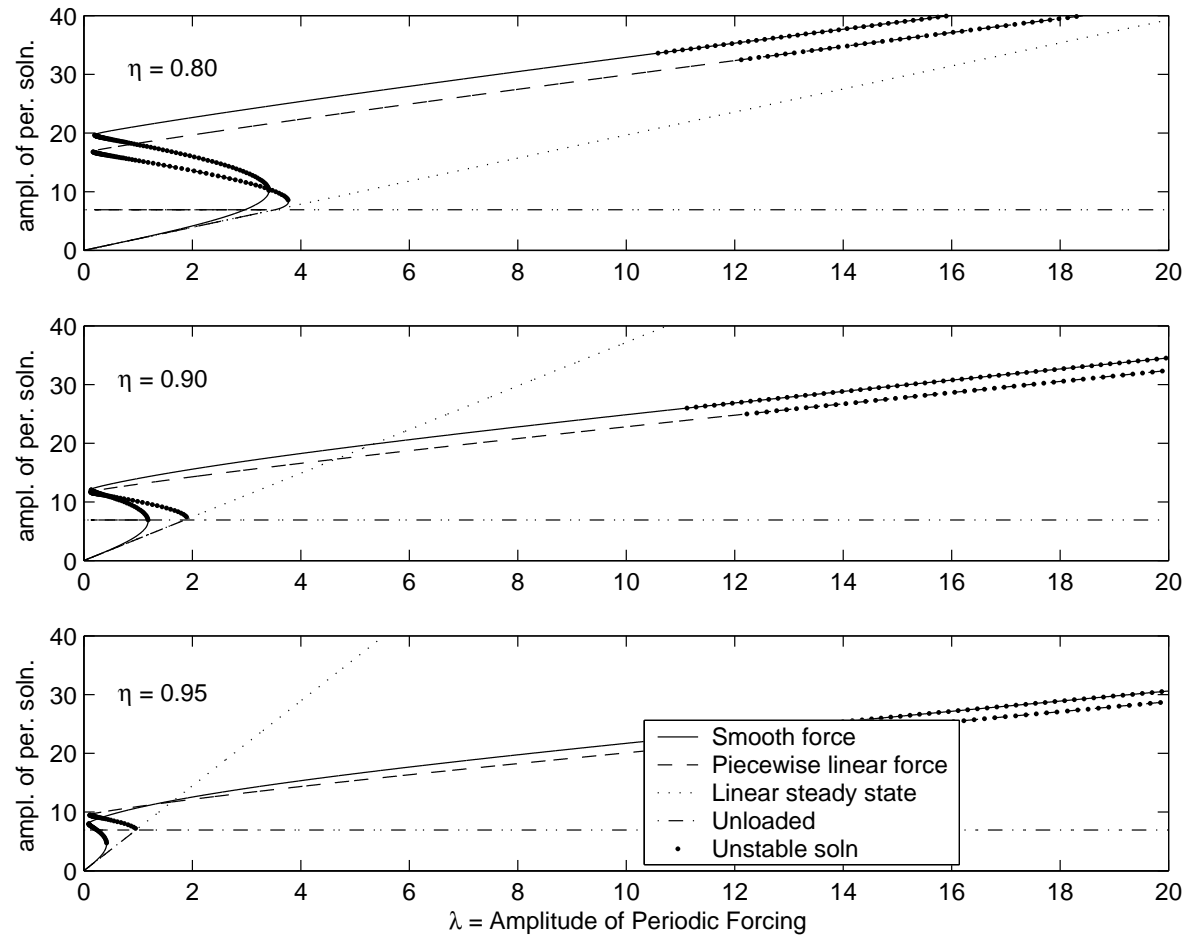

Figure 7: Piecewise and smooth bifurcation curves for varying $\eta$ values 

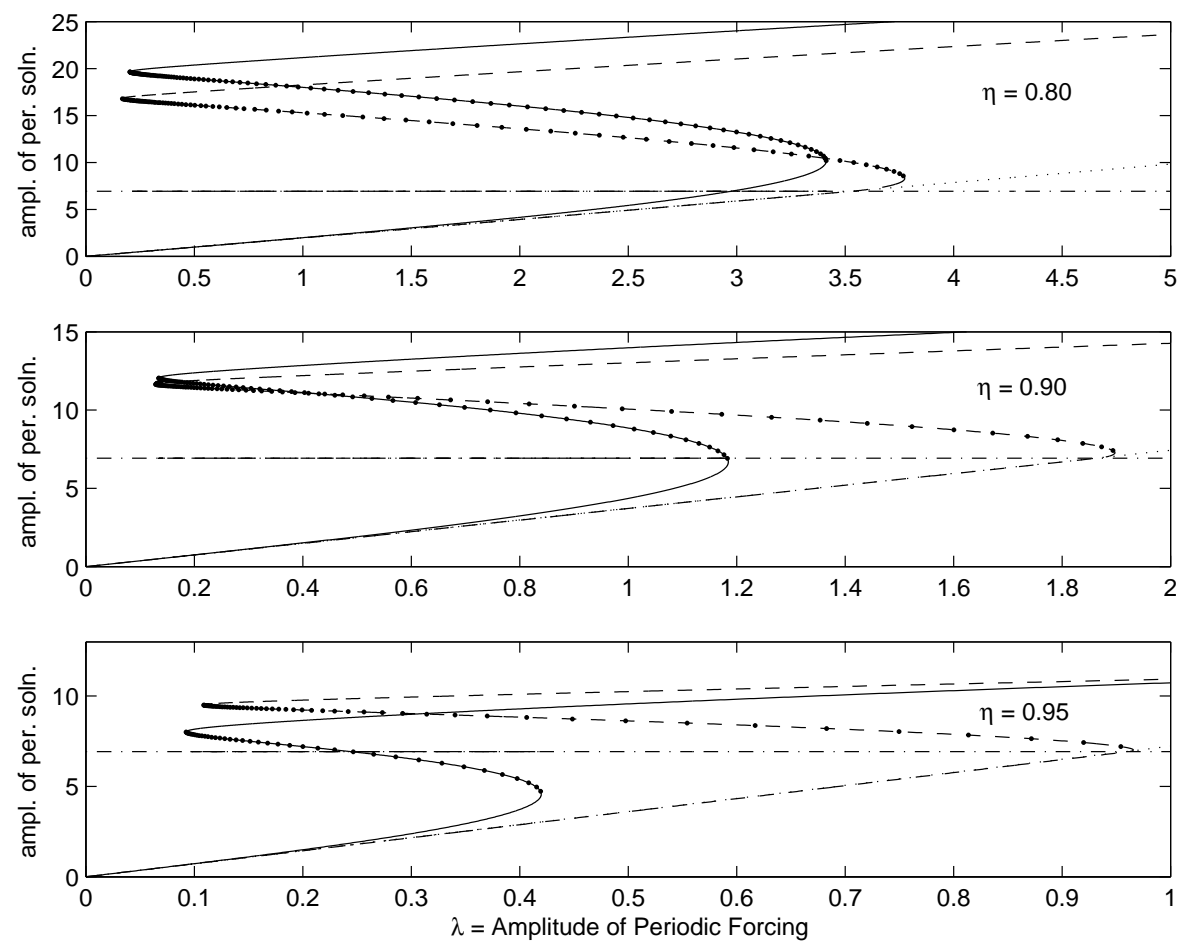

Figure 8: A closeup of the piecewise and smooth bifurcation curves at varying $\eta$ values 
Another aspect which is affected by changes in $\eta$ is the location of the right-hand bifurcation point for the smoothed force. As we saw in Section 3 , for the piecewise linear model, the turning point from the lower to middle branch occurs when the amplitude reached $L$; i.e., when the cable became unloaded. This happens regardless of the forcing frequency $\mu$. In Figure 8, for all $\eta$, we see that the smooth curve diverges from the linear steady state and piecewise curves at a smaller amplitude than $L$ and that when $\eta=0.8$, the turn from the lower branch to the middle branch occurs at higher amplitude than $L$. In fact, the smooth curve turns just before it crosses the piecewise curve. This was true for all values of $K$ that we tested.

When $\eta=0.9$, the smooth curve turns exactly when it hits an amplitude of $L$, and for $\eta=0.95$ it turns before the amplitude $L$. The piecewise curve's behavior is notably different. The piecewise curve always turns into the middle branch at or slightly above amplitude $L$.

In addition, for $\mu$ significantly far from resonance (smaller $\eta$ ), the linear steady state is smaller than both the piecewise and smooth solutions. This is evidenced in Figure 8 for $\eta=0.8$. As we increase $\eta$ towards 1 , we see that eventually the linear steady state can become larger than both the piecewise and smooth curves, and that as we get increasingly close to resonance, the $\lambda$ necessary for the linear steady state to exceed the piecewise and smooth curves becomes smaller. This is because the amplitude of the linear steady state increases more rapidly with $\lambda$ (i.e., the slope of the dotted line in Figure 8 is greater) when $\mu$ is closer to $\mu_{\text {res }}$.

Finally, as $\eta$ approaches 1 , the range of $\lambda$ for which we have three periodic solutions decreases, and is always smaller for the smooth bifurcation curve.

\subsection{Impact of varying $\mathrm{K}$}

While there are multiple notable implications of varying $\eta$, the results of varying $K$ are less pronounced. In Figure 9, we fix $m=1, \delta=.01, \eta=$ $0.9, \mu=1.0703$ and examine the impact of varying $K$. The most notable difference is that the amplitude of the oscillations decreases as $K$ increases. This is logical, as it means that a more rigid cable has a smaller range of motion. For larger $K$ (i.e., for a more rigid cable), the bifurcation from one to three periodic solutions occurs for smaller forcing amplitude $\lambda_{l}$, as does the bifurcation from three to one periodic solutions $\lambda_{u}$. The change in value for $\lambda_{l}$ is more noticeable than the change for $\lambda_{u}$ though. 

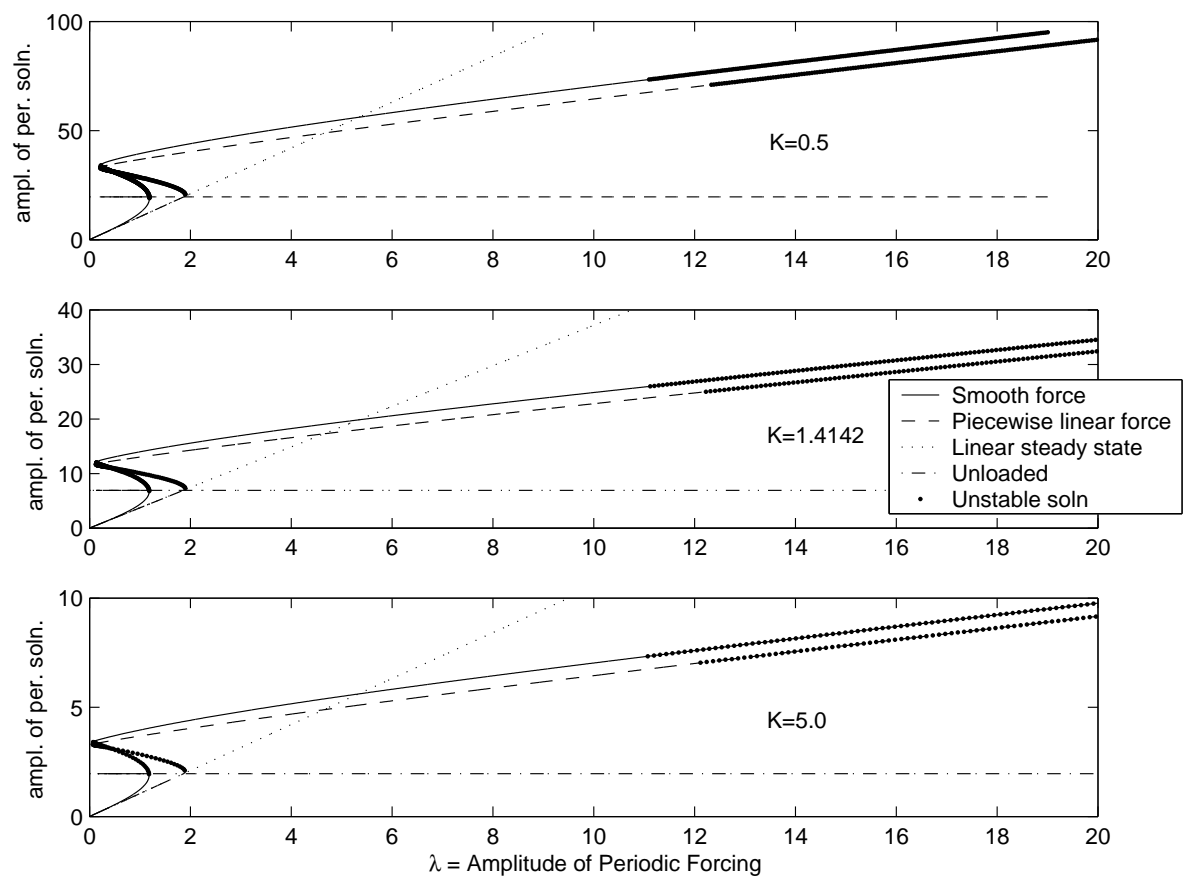

Figure 9: Piecewise and smooth bifurcation curves for varying values of $K$ 


\section{Secondary bifurcations: Period doubling, quadrupling, and chaos}

In previous sections we addressed only periodic solutions whose period matched that of the forcing term, $\tau=\frac{2 \pi}{\mu}$; all the bifurcation curves consisted of only those solutions with period $\frac{2 \pi}{\mu}$. In this section we investigate those unstable solutions that settle down to periods that are double or quadruple the period of the forcing term, and those that do not settle at all, but instead descend into chaos. We will investigate the Poincare' return map of one such solution to illustrate its chaotic nature.

We will examine these secondary bifurcations for $\eta=0.90, m=1, K=$ $\sqrt{2}, \delta=.01$, and $\mu=1.0703$. To do so, we choose an unstable solution on the top branch of the $1 \tau$-periodic curve, identify its long term behavior, which is a $2 \tau$-periodic solution, and then use numerical continuation to find the path of $2 \tau$-periodic solutions. We generate the path of $4 \tau$-periodic solutions the same way. The $2 \tau$-periodic curves obtained by the piecewise linear and smoothed nonlinear models are similar, as you can see in Figure 10 and Figure 11. The curves have approximately the same shape, but the smoothed curve extends noticeably higher and further than the piecewise curve, providing us with solutions at higher $\lambda$ values which the piecewise curve does not.

Much more apparent are the stark differences between the bifurcation curves of period $4 \tau$. As you can see in Figure 10 and Figure 11, the shapes of the curves are dramatically different. Most notably, the range of $\lambda$ for which we found three $4 \tau$ periodic solutions is considerably larger for the smoothed system than for the piecewise system. In addition, the smoothed $4 \tau$-periodic bifurcation curve joins the smoothed $2 \tau$-periodic curve at the site of our chosen starting solution. In stark contrast, the piecewise $4 \tau$-periodic curve appears to extend from the $2 \tau$-periodic piecewise curve, i.e. the $4 \tau$-periodic curve starts where the $2 \tau$-periodic curve ends. Also, the $4 \tau$-periodic piecewise curve quickly reaches amplitudes more than twice as high as those seen in the $2 \tau$-periodic piecewise curve, while the smoothed $4 \tau$-periodic curve is actually smaller in many places than its $2 \tau$-periodic counterpart. In addition, the piecewise curve extends to significantly higher values of $\lambda$ than the smoothed curve achieves, the reverse of what was seen in the $2 \tau$-periodic curves.

Another notable difference is the shape of the solutions found in the bifurcation curves of the respective models. The piecewise and smoothed 


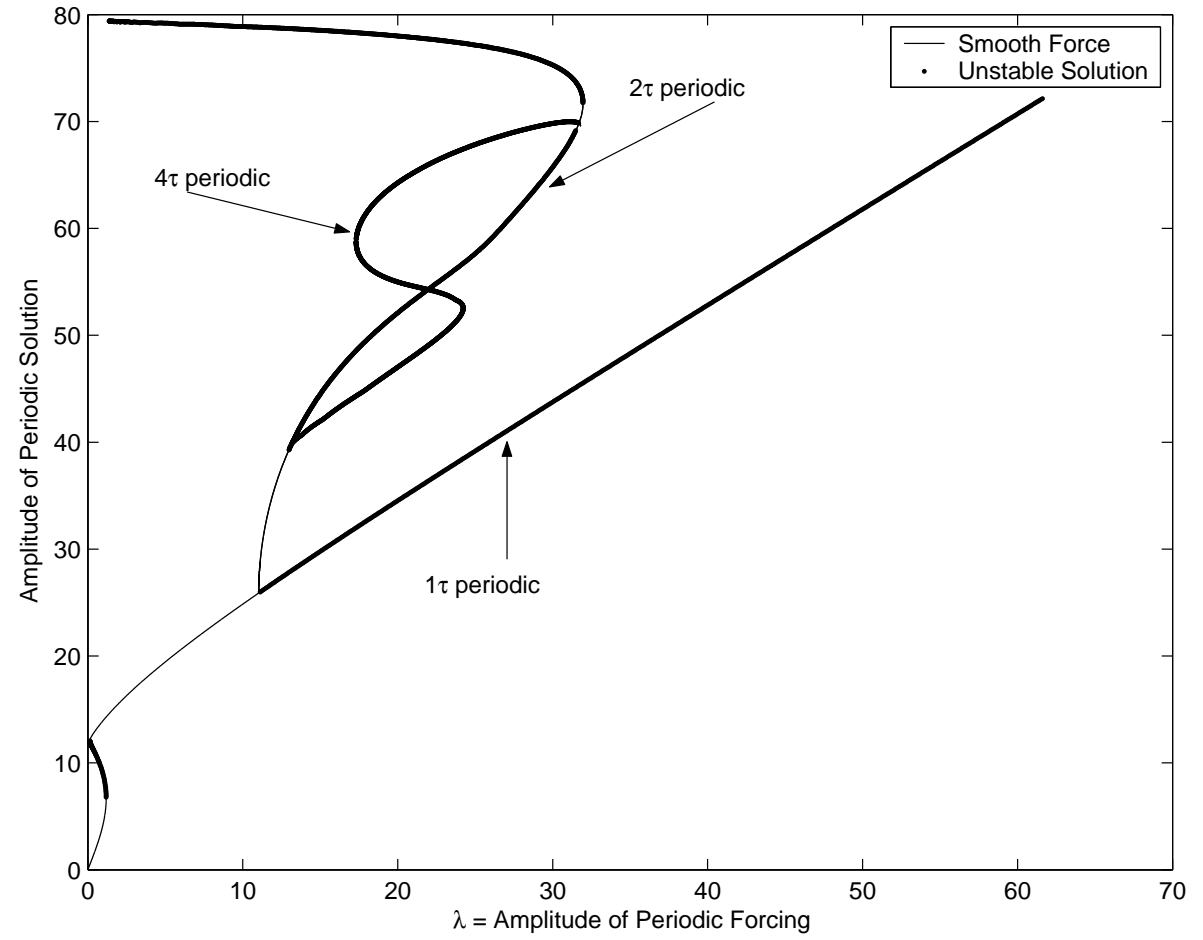

Figure 10: Secondary bifurcations for the smoothed system 


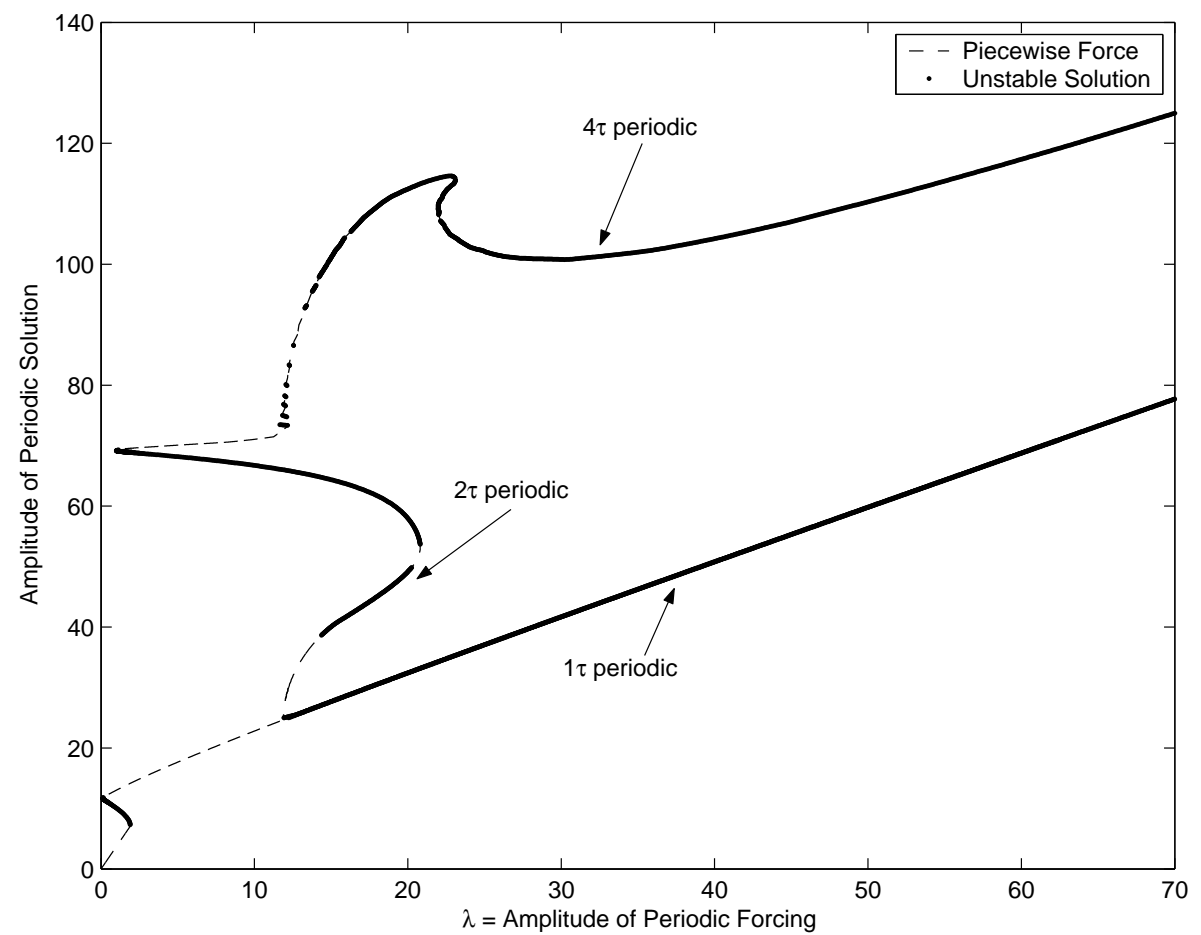

Figure 11: Secondary bifurcations for the piecewise system 

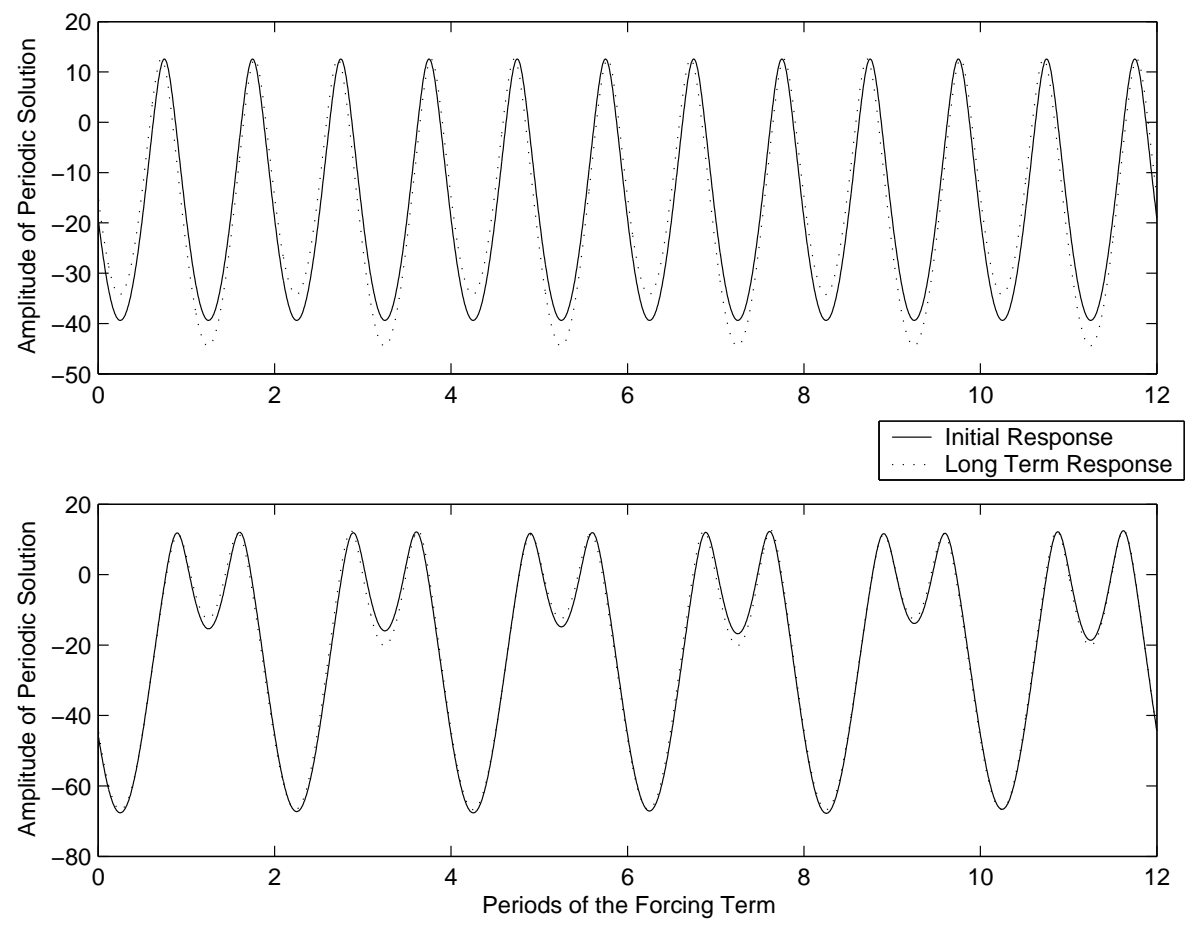

Figure 12: Unstable solutions on smoothed bifurcation curve transitioning form periods $1 \tau$ to $2 \tau$ above, and $2 \tau$ to $4 \tau$ below

models show a similar transformation from the unstable $1 \tau$-periodic to the stable $2 \tau$-periodic curves, as can be seen in the top graphs of Figure 12 and Figure 13. The only apparent difference in this transition is that the piecewise period doubling provides an amplitude change of slightly higher magnitude.

The change in the smooth solution from the unstable $2 \tau$-periodic to the stable $4 \tau$-periodic is slight and the new solution retains the approximate shape of the $2 \tau$-periodic solutions. The piecewise $4 \tau$-periodic solution, on the other hand, is vastly different from the piecewise $2 \tau$-periodic solution, as shown in the second graph in Figure 13. The amplitude nearly doubles and the shape of the curve is dramatically different.

In Figure 14, we examine the Poincare' return map for a chaotic solution to the piecewise model. We begin with an unstable solution that is close to the $2 \tau$-periodic unstable solution that we examined in Figure 13; however, this solution does not settle down to periodic motion as the one in Figure 13 

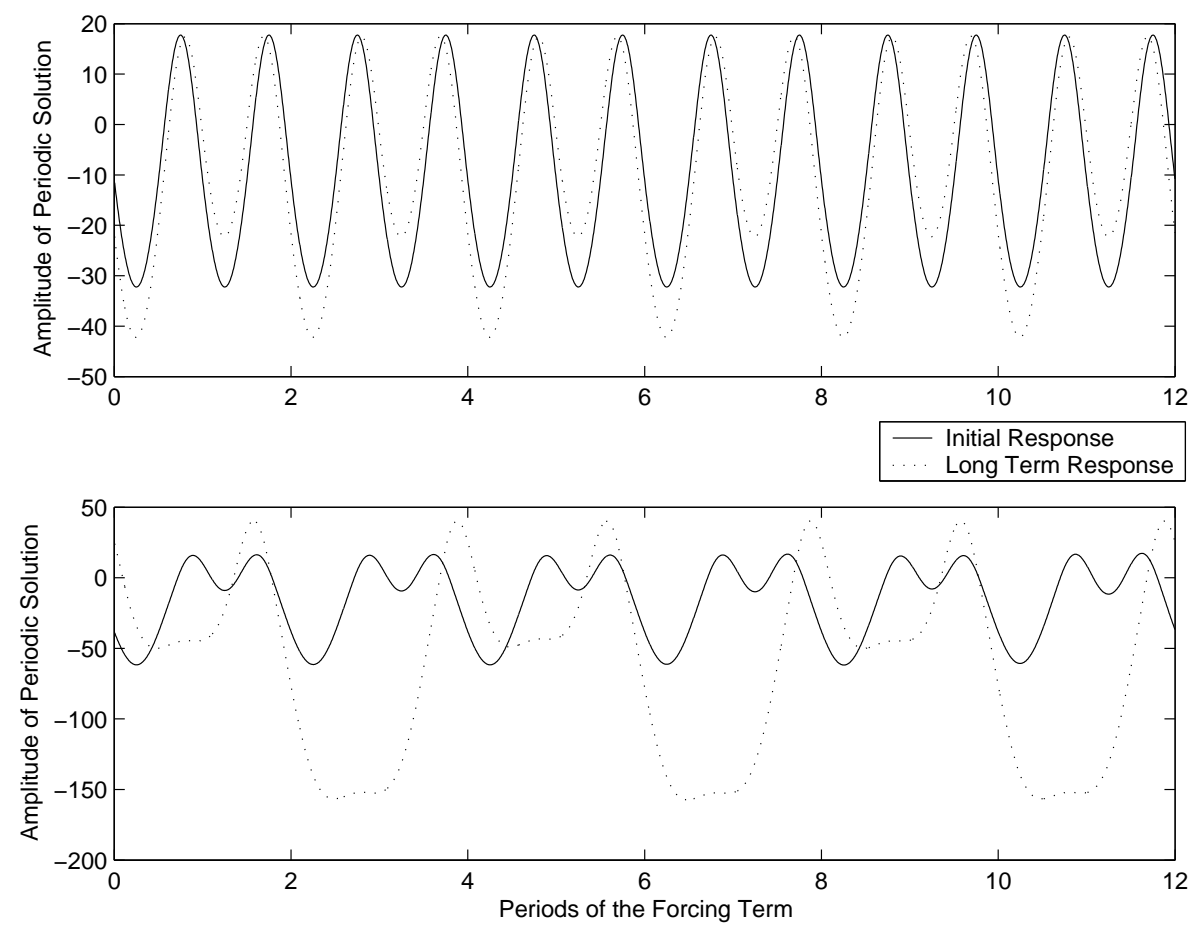

Figure 13: Unstable solutions on piecewise bifurcation curve transitioning form periods $1 \tau$ to $2 \tau$ above, and $2 \tau$ to $4 \tau$ below 


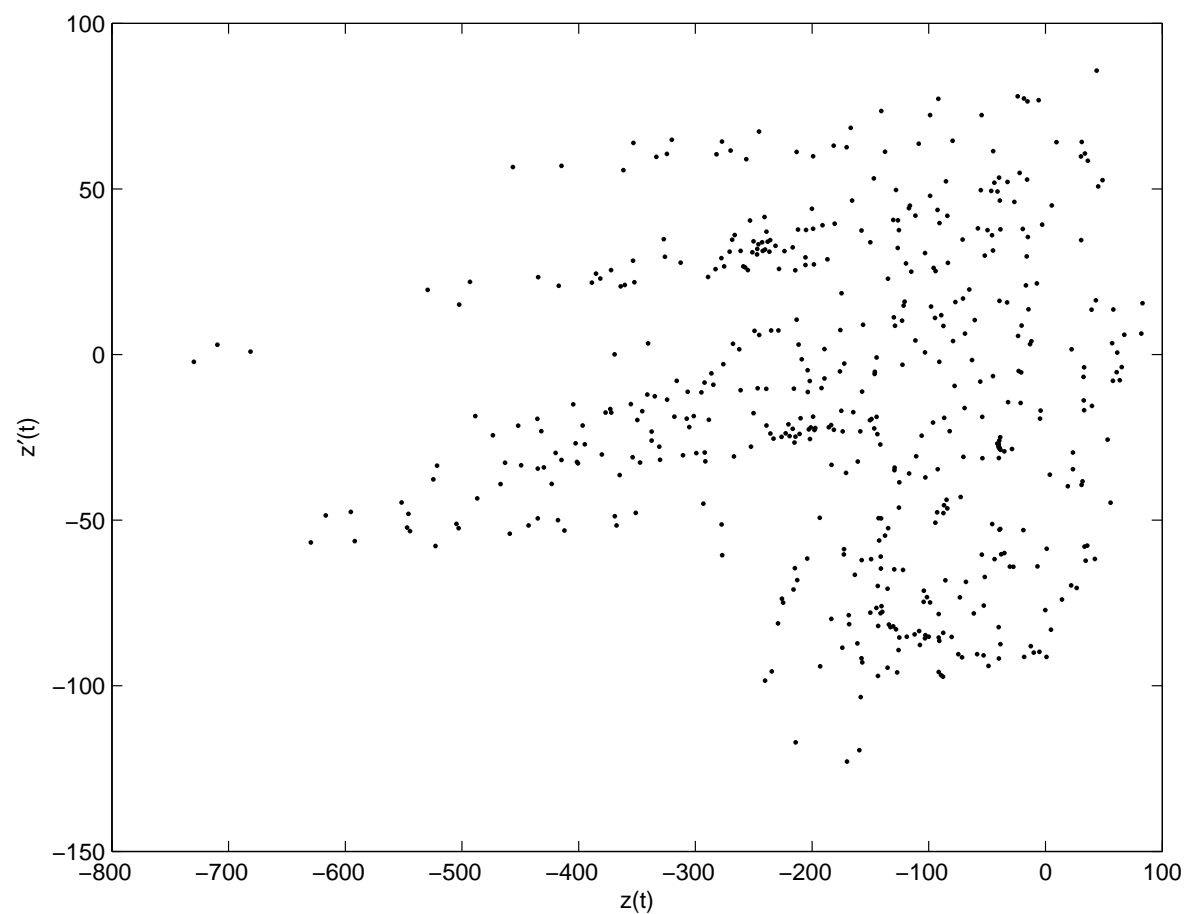

Figure 14: Poincare map for unstable $2 \tau$-periodic solution on the piecewise bifurcation curve

did. We solved the initial value problem for 1000 periods of the forcing term. Figure 14 shows $\left(z\left(t_{n}\right), z^{\prime}\left(t_{n}\right)\right)$ for $t_{n}=2 n \tau, n=0,1,2 \ldots$ As Figure 14 shows, there is no clear pattern and the solution is chaotic.

\section{Concluding Remarks and Open Questions}

In this paper, we identified several phenomena that are consistent with our physical and mathematical intuition; see Section 4.2, for example. But we also identified phenomena for which the mathematical and physical intuition are unclear. For example, in Section 4.1, we saw that when $\eta=0.8$, the first turn in the smooth bifurcation curve occurs exactly as this curve crosses the piecewise curve. When $\eta=0.9$, the first turn in the smooth curve occurs as this curve crosses the height $L$. When $\eta=0.95$, the first turn is at a height lower than $L$. This pattern appeared for every value of $K$ that we tested. Is there a mathematical or physical explanation for this apparent relationship 
between the forcing frequency and the amplitude of the bifurcation points for the two systems?

In Section 5 we examined the nature of the secondary bifurcations for the two systems. We observed that the shape of the $2 \tau$ and $4 \tau$ periodic branches differs significantly. Moreover, though both systems had period doubling and quadrupling, the change in the nature of the solutions was subtle for the smoothed problem and pronounced for the piecewise system. (See Figures 12 and 13.) What is the mathematical underpinning for these phenomena?

\section{References}

[1] Blanchard, Devaney, and Hall. Differential Equations. Brooks/Cole Publishing Company, Pacific Grove, 1998.

[2] Chen, Y. and McKenna, P. J. Traveling waves in a nonlinearly suspended beam: some computational results and four open questions. Localization and solitary waves in solid mechanics, 379-388, Adv. Ser. Nonlinear Dynam., 12, World Sci. Publishing, River Edge, NJ, 1999.

[3] Coddington, E. A. and Levinson, N. Theory of Ordinary Differential Equations. McGraw-Hill, New York, 1955.

[4] Doole, S. H. and Hogan, S. J. Non-linear dynamics of the extended LazerMcKenna bridge oscillation model. Dyn. Stab. Syst., 15 (2000), no. 1, 43-58.

[5] Choi, Y. S., Jen, K. C., and McKenna, P. J. The structure of the solution set for periodic oscillations in a suspension bridge model. IMA J. Appl. Math. 47 (1991), no. 3, 283-306.

[6] Humphreys, L. D. and McKenna, P. J. Multiple periodic solutions for a nonlinear suspension bridge equation. IMA J. Appl. Math. 63 (1999), no. $1,37-49$.

[7] Keller, H. B. Lectures on Numerical Methods in Bifurcation Problems. Springer-Verlag, Berlin, 1987. 
[8] Lazer, A. C. and McKenna, P. J. Large-amplitude periodic oscillations in suspension bridges: some new connections with nonlinear analysis. SIAM Rev. 32 (1990), no. 4, 537-578.

[9] Lazer, A. C. and McKenna, P. J. Large scale oscillatory behaviour in loaded asymmetric systems. Ann. Inst. H. Poincare Anal. Non Lineaire 4 (1987), no. 3, 243-274.

[10] McKenna, P. J. Large torsional oscillations in suspension bridges revisited: fixing an old approximation. Amer. Math. Monthly, 106 (1999), no. $1,1-18$.

[11] McKenna, P. J. and O'Tuama, C. Large torsional oscillations in suspension bridges visited again: vertical forcing creates torsional response. Amer. Math. Monthly 108 (2001), no. 8, 738-745.

[12] McKenna, P. J. and Moore, K. S. The global structure of periodic solutions to a suspension bridge mechanical model. IMA J. Appl. Math. 67 (2002), no. 5, 459-478.

[13] McKenna, P. J. and Walter, W. Nonlinear oscillations in a suspension bridge. Arch. Rational Mech. Anal. 98 (1987), no. 2, 167-177.

[14] Moore, K. S. Large torsional oscillations in a suspension bridge: multiple periodic solutions to a nonlinear wave equation. SIAM J. Math. Anal. 33 (2002), no. 6, 1411-1429.

[15] Scott, A. The Encyclopedia of Nonlinear Science. Routledge, New York, 2004. 